\title{
V7 Elaboração de material de referência in house para vacina de Haemophilus influenzae tipo B e seus produtos intermediários
}

\author{
Elô de Oliveira Rodrigues ${ }^{1}$, Jéssica Yukie dos Reis Nagashima² \\ ${ }^{1}$ ENSP, Fiocruz, RJ \\ 2 Bio-Manguinhos, Fiocruz, RJ
}

Introdução: De acordo com a norma ABNT NBR ISO/IEC 17025:2005, o laboratório deve estabelecer um sistema da qualidade apropriado ao escopo das suas atividades. A utilização de materiais de referência (MR) confere uma confiabilidade junto a outras ações, tais como calibração e qualificação de equipamentos e validação de métodos analíticos. A aquisição de MR ou padrões que possuam características semelhantes aos produtos biológicos é uma das dificuldades encontradas pelos laboratórios de controle de qualidade de vacinas. Os poucos MR existentes são de custo elevado e com pouca quantidade disponível para aquisição, tornando-os inviáveis para a utilização em análises de rotina.

Objetivo: Estabelecer a metodologia de produção de material de referência in house para os ensaios físico-químicos do controle de qualidade dos produtos obtidos em Bio-Manguinhos.

Metodologia: Para este trabalho utilizamos dois produtos intermediários, o polirribosil-ribitol fosfato (PRRP) e o PRRP conjugado com a proteína monomérica tetânica (PSTT), que fazem parte da etapa de produção da vacina de Haemophilus influenzae tipo b. A elaboração destes dois MR in house foram realizados de acordo com os guias ISO 30-35, que estabelecem os parâmetros e condições para a sua produção, sendo rastreáveis à MR internacional NIBSC e outros, como Pierce e D'ribose, que aumentam a confiabilidade dos MR produzidos. $\mathrm{O}$ uso dos MR in house se aplica às seguintes análises: determinação de fósforo (Método de CHEN), determinação de D'ribose (método de Bial) e determinação de proteína (Método de Lowry). As etapas para elaboração foram: caracterização, homogeneidade e estabilidade real e acelerada. Os resultados foram avaliados através de ferramentas estatísticas ANOVA e análise de tendência por regressão linear.

Resultados: Todos os parâmetros de aceitação dos MR foram atendidos. A homogeneidade dos dois MR apresentou Fcalculado $<$ Fcrítico e valor de $\mathrm{p}<0,05$, confirmando a homogeneidade dos resultados entre frascos e intra-frascos para $95 \%$ de significância. As avaliações dos estudos de estabilidade a longo prazo e acelerada através da análise de tendência por regressão linear atenderam à condição estabelecida para estabilidade lb1l<t0,95, n-2.s(b1). Através das etapas de elaboração dos MR obteve-se os componentes de incerteza da caracterização, homogeneidade e estabilidade, gerando a incerteza combinada dos materiais de referência para cada método. 
Para o MR in house PRRP, os valores estabelecidos são: D'ribose $191,12 \pm 11,74 \mu \mathrm{g} m \mathrm{~mL}$ e Fósforo $36,33 \pm 2,46 \mu \mathrm{g} / \mathrm{mL}$. Já para o MR in house PSTT os valores estabelecidos são: D'ribose 878,75 \pm $40,80 \mu \mathrm{g} / \mathrm{mL}$ e proteína $1314,54 \pm 45,48 \mu \mathrm{g} / \mathrm{mL}$.

Conclusão: Conclui-se que os MR in house produzido atendem aos propósitos preestabelecidos, em conformidade com as normas vigentes. Devido à sua abrangência, estes materiais atendem ao controle de qualidade dos seguintes produtos: PRRP, PSTT e vacina Hib sendo utilizados no controle físico-químico de Bio-Manguinhos e por outros laboratórios, como o Instituto Nacional de Controle de Qualidade em saúde (INCQS).

Palavras-Chave: Material de Referência, Cálculos Incerteza 